\title{
RECORRÊNCIA PRECOCE DE NEFROPATIA DIABÉTICA PÓS-TRANSPLANTE RENAL - RELATO DE CASO E REVISÃO DA LITERATURA
}

\author{
Early recurrence of diabetic nephropathy in kidney recipients \\ - case report and literature review
}

Beatriz de Oliveira Neri, Adriana Regina Vilarinho de Oliveira, Paula Frassinetti Castelo Branco Camurca Fernandes, Claudia Maria Costa de Oliveira

\section{RESUMO}

A nefropatia diabética (ND) é atualmente a principal causa da doença renal em estágio terminal (DRET), na Europa e Estados Unidos, e a segunda causa, no Brasil. A recorrência histológica da nefropatia diabética tem sido descrita em cerca de $40 \%$ dos pacientes, sendo o diagnóstico de recorrência realizado em média de 6-8 anos pós-transplante renal, mas, com relatos de recorrência tão precoce como dois anos. Relatamos o caso de um paciente com diagnóstico prévio de diabetes mellitus que foi submetido a transplante renal com doador falecido e evoluiu à recorrência de nefropatia diabética, no primeiro ano após transplante, com diagnóstico de certeza, somente após biópsias repetidas.

Descritores: Transplante de Rim, Recorrência, Diabetes Mellitus

\section{Instituição:}

Departamento de Transplante Renal do Hospital Universitário Walter Cantídio da Universidade Federal do Ceará, Fortaleza/CE, Brasil

\section{Correspondência:}

Beatriz de Oliveira Neri

beatrizneri@gmail.com

https://doi.org/10.53855/bjt.v24i1.008

\section{INTRODUÇÃO}

A nefropatia diabética (ND) é atualmente a principal causa da doença renal em estágio terminal (DRET), na Europa e Estados Unidos ${ }^{1-7}$ e a segunda causa, no Brasil. ${ }^{8} \mathrm{O}$ Censo da Sociedade Brasileira de Nefrologia (2018) estima prevalência de $31 \%$ de nefropatia diabética como etiologia da DRET em hemodiálise. ${ }^{8} \mathrm{O}$ transplante renal é o tratamento de escolha nesses casos, uma vez que confere melhor sobrevida ao paciente. ${ }^{3} \mathrm{Um}$ estudo americano analisou a sobrevida de aproximadamente 230.000 pacientes em diálise, utilizando dados do United States Renal Data System (USRDS). Nessa coorte, 46.000 pacientes foram listados para transplante renal e 23.000 foram submetidos a transplante renal com doador falecido. A sobrevida foi significativamente 
maior entre pacientes transplantados, em comparação aos que permaneceram em lista de espera, sendo essa maior sobrevida também evidenciada na subpopulação de pacientes com diagnóstico de diabetes (DM). ${ }^{9}$

Existem poucos estudos que abordam a recorrência da ND pós- transplante renal e a maioria não faz diferença entre pacientes com diabetes tipo 1 ou $2 .^{3}$ Além disso, a maioria dos estudos em ND pós-transplante não diferencia entre DM pré-existente e DM de novo póstransplante, cuja incidência varia de $4-25 \%$. $^{3,10}$

A recorrência histológica da ND tem sido descrita em cerca de $40 \%$ dos pacientes com diagnóstico prévio de diabetes, ${ }^{11}$ ocorrendo, em média, 6-8 anos póstransplante, mas com relatos de recorrência precoce em dois anos. ${ }^{3-5,10,12-15}$

Os fatores clínicos e genéticos que predispõem à ND nos rins nativos são bem estudados e conhecidos, como a duração da diabetes, pressão arterial elevada, controle glicêmico inadequado, hiperlipidemia, obesidade e fenótipos HLA (A30, B8, B15, B27, B42 E DR4), entre outros. Por outro lado, há ainda poucos dados em relação à recorrência de nefropatia diabética em enxertos renais. ${ }^{3,10}$

Os autores apresentam o caso clínico de um paciente com DM tipo 2 pré-transplante, que evoluiu com recorrência da ND no enxerto renal no primeiro ano póstransplante, comprovada por biópsia renal.

\section{Relato do caso clínico}

Paciente masculino, 62 anos, portador de doença renal crônica por DM tipo 2, insulinodependente há 23 anos, em hemodiálise há cinco anos, IMC $23,4 \mathrm{~kg} / \mathrm{m} 2$, PRA (anticorpos reativos contra painel de linfócitos) classe I de $62 \%$ e classe II de $0 \%$, respectivamente. Foi submetido a transplante renal com doador falecido, masculino, 52 anos, causa da morte encefálica traumatismo crânio encefálico, IMC 23,4 kg/m2, sem história clínica de diabetes, com creatinina inicial de $0,7 \mathrm{mg} / \mathrm{dl}$ e final de $2,0 \mathrm{mg} / \mathrm{dl}$, com 5 mismatches HLA. A biópsia renal do doador, no momento da captação, revelou: necrose tubular aguda leve, fibrose intersticial leve e difusa, arterioloesclerose hialina leve, microtrombos nos capilares glomerulares e algumas arteríolas, além de aumento discreto da matriz mesangial em alguns glomérulos. O enxerto renal foi preservado em máquina de perfusão com tempo de isquemia fria (TIF) de $22 \mathrm{~h}$ e 40 minutos (perfusão estática: $6 \mathrm{~h}$ 8min e dinâmica: 15h10min).

O paciente apresentava anticorpo específico contra o doador (DSA) anti-A31 com MIF (intensidade média de fluorescência) de 1146, sendo realizado protocolo de indução com timoglobulina e manutenção com tacrolimo e micofenolato sódico, sem uso de corticoide. Evoluiu com função retardada do enxerto (DGF), tendo realizado hemodiálise até $013^{\circ}$ pós-operatório, no total de sete sessões de diálise. Recebeu alta hospitalar no $20^{\circ}$ dia pós-transplante, com creatinina de $3,2 \mathrm{mg} / \mathrm{dl}$, chegando depois a níveis normais de $0,8 \mathrm{mg} / \mathrm{dl}$.

Após oito meses de transplante, foi detectada proteinúria inicial de $1020 \mathrm{mg} / 24$ horas, com piora progressiva nos meses subsequentes, mesmo em uso regular de losartana em dose otimizada, porém sem alterações nos níveis de lipídeos e albumina.

Uma biópsia renal foi realizada aos 10 meses, com creatinina de $0,8 \mathrm{mg} / \mathrm{dl}$ (TFGe $96 \mathrm{ml} / \mathrm{min}$ ), PTN de 3,8g/24 hs e nível sanguíneo de tacrolimo $7 \mathrm{ng} / \mathrm{ml}$, com microscopia ótica (MO) mostrando necrose tubular aguda (NTA) leve e glomerulonefrite proliferativa mesangial, com C4d negativo, imunofluorescência (IMF) com C3c positivo $(+/ 3+)$ e IgM positivo (+/3+). O PRA na ocasião persistia com DSA anti-A31, havendo aumento de MIF para 3661. A avaliação laboratorial revelou dosagem normal de frações do complemento, sorologia para hepatite B, C e HIV negativas, imunoeletroforese de proteínas séricas sem bandas monoclonais e radiografia de ossos longos sem sinais de acometimento por doença monoclonal ou neoplasias.

Uma segunda biópsia renal foi realizada 22 meses póstransplante, com creatinina de $1,1 \mathrm{mg} / \mathrm{dL}$ (TFGe $71 \mathrm{ml} /$ min), PTN de 4,6g/24hs e nível sanguíneo de tacrolimo $6 \mathrm{ng} / \mathrm{ml}$. A MO demonstrou NTA leve, aumento leve da matriz e celularidade mesangiais, hialinose arteriolar e ausência de sinais de rejeição, com C4d negativo e IMF inconclusiva (ausência de glomérulos na amostra). $O$ PRA nesta ocasião não demonstrou DSA.

Após 40 meses do transplante renal, com creatinina de $1,2 \mathrm{mg} / \mathrm{dL}$ (TGFe $64 \mathrm{ml} / \mathrm{min}$ ), PTN de $5,1 \mathrm{~g} / 24$ hs e nível sanguíneo de tacrolimo $4 \mathrm{ng} / \mathrm{ml}$, uma terceira biópsia renal foi realizada, com MO sugestiva de glomerulonefrite mesangioproliferativa, sem espessamento das alças capilares, hialinose arteriolar, discreto espessamento fibroso intimal, ausência de sinais de rejeição, C4d negativo e IMF negativa, segundo o patologista, devendo ser considerado o diagnóstico de glomeruloesclerose diabética difusa (figuras $1 \mathrm{~A}$ e 1B).

O paciente manteve função renal estável ao longo de sete anos de seguimento (creatinina 1,3mg/dl; TFG estimada de $56 \mathrm{ml} / \mathrm{min}$ ), HbA1c 7,8\%, nível sanguíneo de tacrolimo $4 \mathrm{ng} / \mathrm{ml}$ e proteinúria abaixo de $1000 \mathrm{mg} / 24$ horas, após associação de espironolactona ao losartana em doses otimizadas. A evolução das glicemias e 
Beatriz de Oliveira Neri, Adriana Regina Vilarinho de Oliveira, Paula Frassinetti Castelo Branco Camurca Fernandes,

proteinúrias pós-transplante encontra-se nas figuras 2 e 3. Outras comorbidades presentes desde o transplante: retinopatia e vasculopatia periférica diabéticas, além de HAS.

Figura 1 - Biópsia renal do paciente em estudo.

\section{Figura $1 A$}

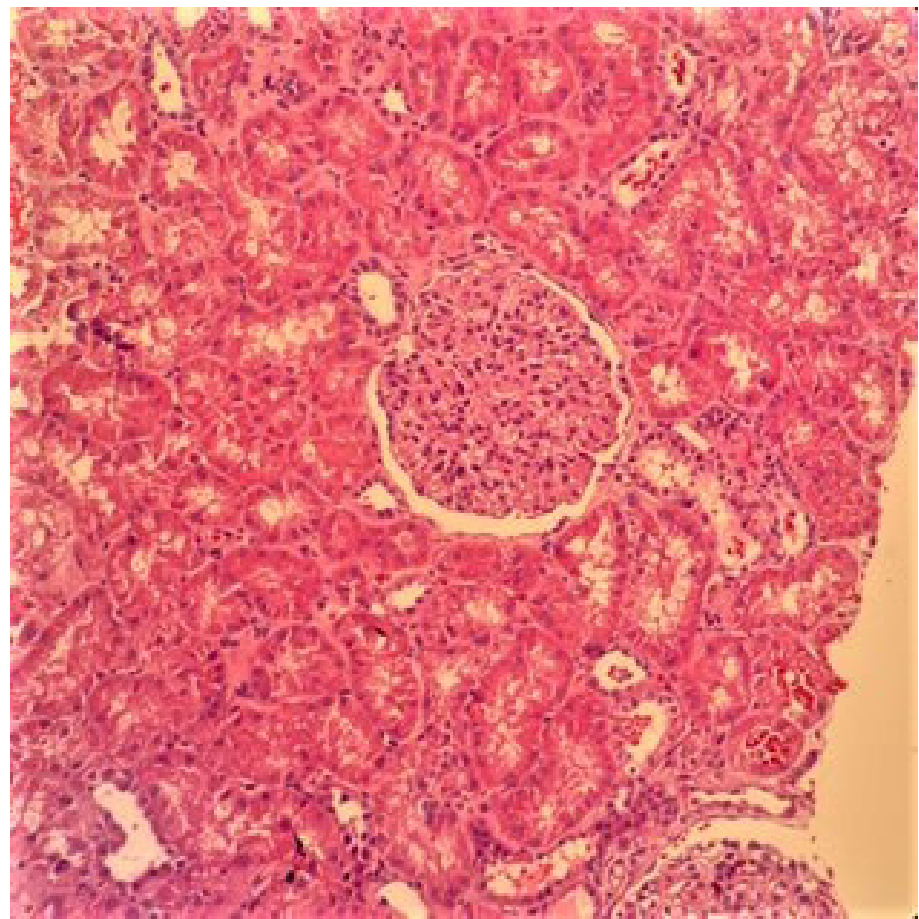

Figura $1 B$

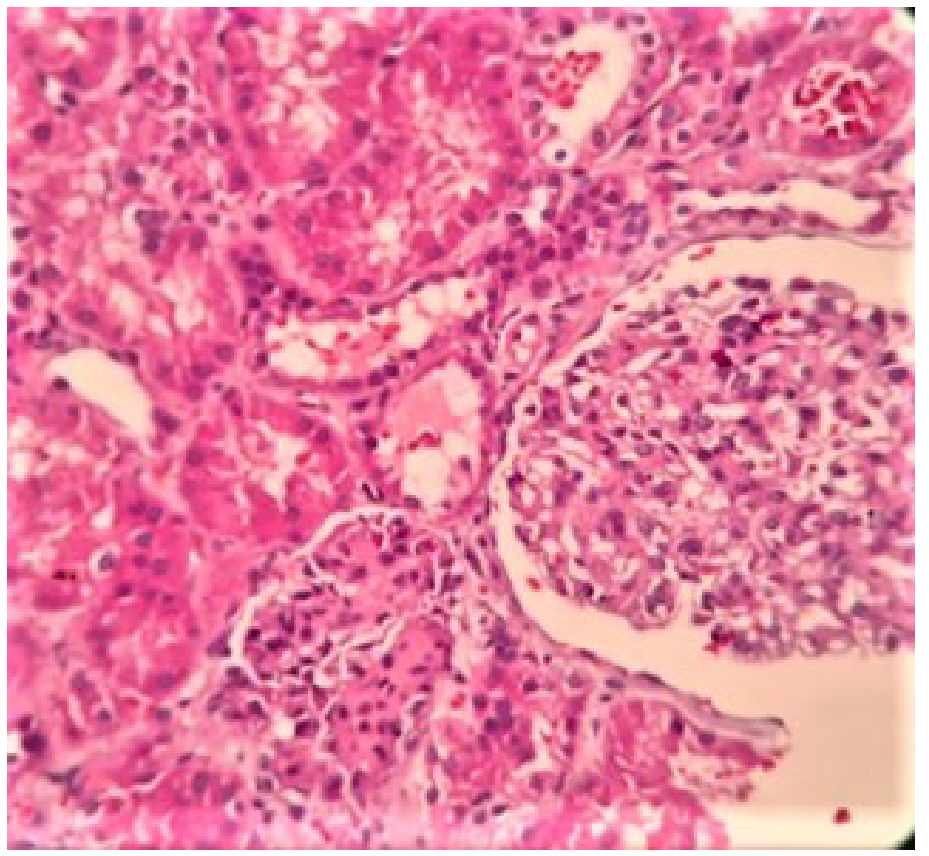

Figura 2 - Evolução da proteinúria de 24 horas pós-transplante

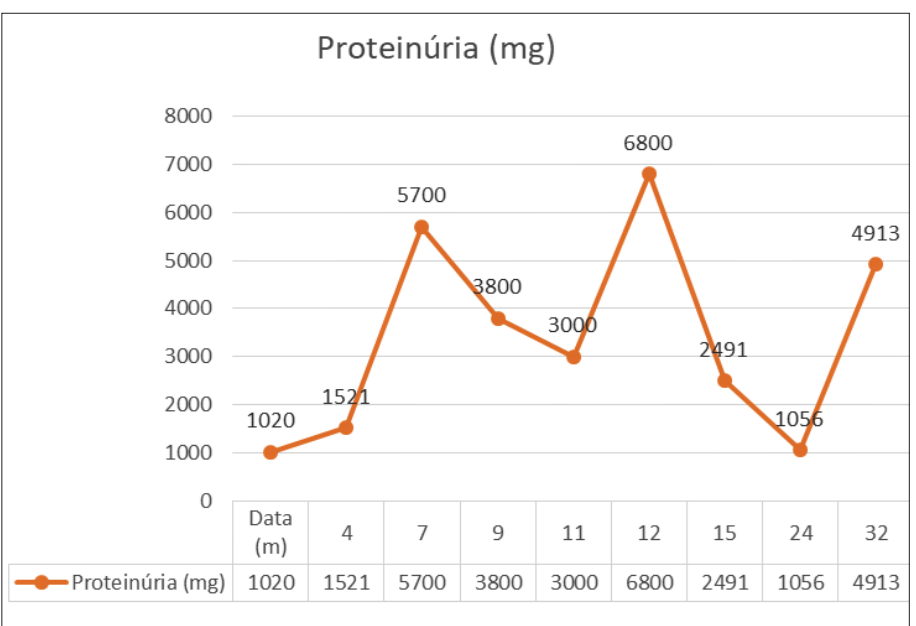

Figura 3 - Evolução da glicemia em jejum pós-transplante.

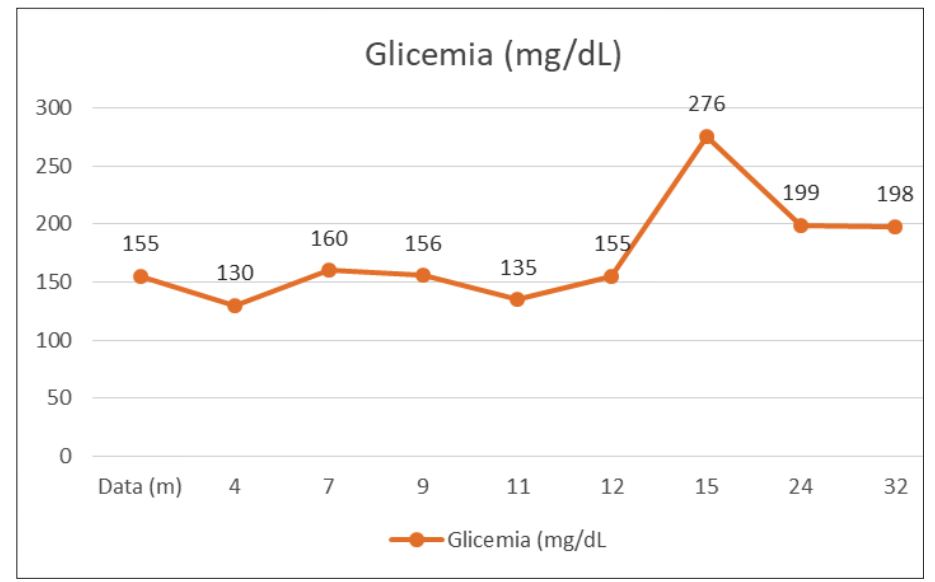

\section{DISCUSSÃO}

No caso descrito, a recorrência de nefropatia diabética após transplante renal, provavelmente ocorreu ainda no primeiro ano de transplante, devido a PTN inicialmente subnefrótica e depois nefrótica, sem outra causa aparente nas biópsias renais, bem como presença de aumento de matriz e celularidade mesangial. $\mathrm{Na}$ literatura, há relatos de alterações em biópsia renal por recorrência de nefropatia diabética, com diagnóstico mais precoce, aos dois anos pós-transplante. ${ }^{12-15}$

Em um estudo retrospectivo, Cimeno et al. avaliaram biópsias renais de 60 pacientes transplantados com DM tipo 2 pré-transplante e a média de tempo do diagnóstico de recorrência da ND foi de 1456 dias ( \pm 206 ), ou seja, algo em torno de quatro anos. Nesse estudo, os fatores que influenciaram o tempo de diagnóstico da 
ND foram o transplante com doador falecido, a idade maior do receptor e a creatinina elevada no momento do diagnóstico. ${ }^{3}$

$\mathrm{Na}$ recorrência da ND, o quadro clínico mais comum é o surgimento de proteinúria, que pode atingir níveis nefróticos, associados à piora lenta da função renal. ${ }^{13,14}$ Apesar das alterações glomerulares precoces, a perda do enxerto renal nos 10 primeiros anos pós-transplante é rara em pacientes com recorrência de ND. ${ }^{15,16}$ No presente estudo, durante aproximadamente sete anos de acompanhamento após transplante renal, o paciente manteve a TFG relativamente estável (com declínio na fase inicial), a despeito da proteinúria.

As principais alterações histopatológicas descritas em biópsias de enxerto renal com ND são expansão e aumento da celularidade mesangial e espessamento da membrana basal glomerular.6,12 Além disso, há alterações vasculares com hialinose de arteríola aferente e eferente e angiogênese glomerular anormal caracterizada por neovascularização do polo vascular glomerular. ${ }^{6,12}$

As biópsias do paciente do presente caso mostravam expansão da matriz e celularidade mesangial, bem como hialinose arteriolar compatíveis com a descrição de ND, embora possa ser questionado que a biópsia do doador no momento da captação apresentava hialinose arteriolar leve e aumento discreto a matriz mesangial, podendo essas alterações serem secundárias à HAS ou DM do doador, que não tinha diagnóstico conhecido destas patologias. Entretanto, as alterações de proteinúria não foram imediatas no receptor, tendo sido detectadas somente após oito meses do transplante. Há relatos em alguns estudos que as alterações mesangiais são as que têm mais correlação com as manifestações clínicas da ND. ${ }^{17}$

O mecanismo associado à ND pós-transplante renal é pouco conhecido, mas acredita-se ser resultado de injúria inicial dos podócitos, levando à posterior progressão para as clássicas alterações da esclerose glomerular diabética e fibrose intersticial, influência dos inibidores da mTOR na função podocitária e/ ou presença de receptores de plasminogênio ativado circulante..$^{3,12}$
Os inibidores da mTOR, largamente utilizados como parte da terapia imunossupressora de manutenção podem ter impacto negativo no reparo glomerular e podocitário, mas com pouca evidência na progressão da nefropatia diabética pós-transplante renal. ${ }^{3,10} \mathrm{O}$ tacrolimo e os inibidores da mTOR são os imunossupressores mais diabetogênicos, mas o tratamento com tacrolimo associa-se a maior risco de recorrência da nefropatia diabética, comparado à ciclosporina, e o uso de estatinas e inibidores do sistema renina-angiotensina-aldosterona podem retardar a progressão da ND. ${ }^{5}$

Há registro de recorrência de ND, tanto em transplantes com doador falecido quanto com doador vivo. Os rins de doador falecido parecem ter maior precocidade de recorrência, provavelmente por fatores relacionados ao doador como idade, história médica, creatinina e TIF, mas há poucos dados na literatura para conclusões robustas. $^{3}$

Hiperglicemia pós-transplante renal parece ser um fator primordial para a recorrência e progressão da ND póstransplante. ${ }^{3,7,10,12} \mathrm{O}$ controle pressórico e lipídico, por outro lado, tem efeito menor no risco de recorrência da nefropatia diabética. ${ }^{12}$ No caso descrito, o paciente apresentou controle glicêmico irregular, com Hba1c em torno de $6,0 \%$ no primeiro ano, 7,0 no segundo ano e $8.0 \%$ no terceiro ano.

\section{CONCLUSÃO}

Pacientes transplantados renais com diagnóstico de diabetes devem ser encorajados a ter estilo de vida saudável, com dieta balanceada e exercícios físicos regulares, além de um ajuste medicamentoso para controle glicêmico adequado. ${ }^{4,5,10-12}$ Inibidores da ECA e bloqueadores do receptor de angiotensina, bem como as estatinas, devem ser utilizados como medicações complementares para controle de proteinúria e diminuição da progressão da nefropatia diabética. ${ }^{5,18}$

O transplante duplo pâncreas-rim tem efeito protetor em relação à recorrência de ND, provavelmente, pela menor incidência de hiperglicemia, devendo ser o tratamento de primeira escolha para pacientes com diabetes mellitus tipo $1.6,7,11,12,18$ 
Beatriz de Oliveira Neri, Adriana Regina Vilarinho de Oliveira, Paula Frassinetti Castelo Branco Camurca Fernandes, Claudia Maria Costa de Oliveira

\section{ABSTRACT}

Diabetic nephropathy (DN) is currently the leading cause of end-stage renal disease (DRET) in Europe and United States and the second leading cause in Brazil. Histological recurrence of diabetic nephropathy has been described in about $40 \%$ of patients, with recurrent diagnosis made on average 6-8 years after kidney transplantation, but with reports of recurrence as early as 2 years. We report the case of a patient with previous diagnosis of diabetes mellitus who underwent kidney transplantation with a deceased donor and developed recurrent diabetic nephropathy in the first year after transplantation, with a diagnosis of certainty only after repeated biopsies.

Keywords: Kidney Transplantation; Recurrence; Diabetes Mellitus

\section{REFERÊNCIAS}

1. Fioretto P, Barzon I, Mauer M. Is diabetic nephropathy reversible? Diabetes Res Clin Pract. 2014 Jun;104(3):323-8.

2. Nefropatia diabética.. https://bjnephrology.org/wp-content/ uploads/2019/11/jbn_v27n1s1a06.pdf. Braz. J. Nephrol. 2005;27(1):32-4.

3. Cimeno A, Munley J, Drachenberg C, Weir M, Haririan A, Bromberg J, et al.. Diabetic nephropathy after kidney transplantation in patients with pretransplantation type II diabetes: A retrospective case series study from a highvolume center in the United States. Clin Transplant. 2018 Dec;32(12):e13425.

4. Bhalla V, Nast CC, Stollenwerk N, Tran S, Barba L, Kamil ES, et al. Recurrent and de novo diabetic nephropathy in renal allografts. Transplantation. 2003 Jan 15;75(1):66-71.

5. Cubillo BR, Rodriguez B, Calvo M, de la Manzanara V, Bautista J, Perez-Flores I, er al. Risk Factors of Recurrence of Diabetic Nephropathy in Renal Transplants. Transplant Proc. 2016 Nov;48(9):2956-8.

6. Fioretto P, Mauer M. Reversal of diabetic nephropathy: lessons from pâncreas transplantation. J Nephrol. 2012 JanFeb;25(1):13-8.

7. Luan FL, Samaniego M. Transplantation in diabetic kidney failure patients: modalities, outcomes, and clinical management. Semin Dial. 2010 Mar-Apr;23(2):198-205.

8. Sociedade Brasileira de Nefrologia. Censo de diálise - 2018. Disponível em https://sbn.org.br/categoria/censo-2018/

9. Wolfe RA, Ashby VB, Milford EL, et al. Comparison of mortality in all patients on dialysis, patients on dialysis awaiting transplantation, and recipients of a first cadaveric transplant. N Engl J Med. 1999;341:1725.

10. Gregorini M, Sepe V, Pattonieri FE, Allesina A, Rampino T. Early onset of graft glomerulopathy in a patient with posttransplant diabetes mellitus after renal transplantation: a case report. BMC Nephrol. 2018;Dec 7;19(1):348.
11. Ponticelli C, Moroni G, Glassock RJ. Recurrence of secondary glomerular disease after renal transplantation. Clin J Am Soc Nephrol. 2011 May;6(5):1214-21.

12. Nyumura I, Honda K, Tanabe K, Teraoka S, Iwamoto Y. Early histologic lesions and risk factors for recurrence of diabetic kidney disease after kidney transplantation. Transplantation. 2012 Sep 27;94(6):612-9.

13. Nyumura I, Honda K, Babazono T, Horita S, Murakami T, Fuchinoue S, et al. Recurrence of diabetic kidney disease in a type 1 diabetic patient after kidney transplantation. Nephrology (Carlton). 2015 Jul;20 Suppl 2:90-2.

14. Pavan M, Ranganath R, Chaudhari AP. Early recurrence of diabetic nodular sclerosis in a kidney transplant recipient. Iran J Kidney Dis. 2012 May;6(3):219-21.

15. Mauer SM, Barbosa J, Vernier RL, Kjellstrand CM, Buselmeier TJ, Simmons RL, Najarian JS, Goetz FC. Development of diabetic vascular lesions in normal kidneys transplanted into patients with diabetes mellitus. N Engl J Med. 1976 Oct 21;295(17):916-20.

16. Owda AK, Abdallah AH, Haleem A, Hawas FA, Mousa D, Fedail H, Al-Sulaiman $\mathrm{MH}, \mathrm{Al}-\mathrm{Khader}$ AA. De novo diabetes mellitus in kidney allografts: nodular sclerosis and diffuse glomerulosclerosis leading to graft failure. Nephrol Dial Transplant. 1999 Aug;14(8):2004-7.

17. Barbosa J, Steffes MW, Sutherland DE, Connett JE, Rao KV, Mauer SM. Effect of glycemic control on early diabetic renal lesions. A 5-year randomized controlled clinical trial of insulin-dependent diabetic kidney transplant recipients. JAMA. 1994 Aug 24-31;272(8):6006.

18. Hariharan S, Smith RD, Viero R, First MR. Diabetic nephropathy after renal transplantation. Clinical and pathologic features. Transplantation. 1996 Sep $15 ; 62(5): 632-5$. 